\title{
Critères qualité: performants et faciles à implémenter
}

\author{
Jürg Schlup \\ Dr méd., président de la FMH
}

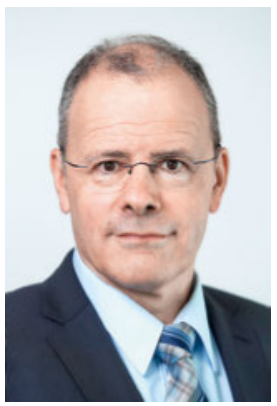

La limitation actuelle de l'admission des médecins arrive à échéance dans un peu plus de deux ans, si bien que les discussions sur les nouvelles options de pilotage sont déjà bien avancées. D'ici quelques semaines, le Conseil fédéral présentera un rapport pour répondre au postulat 16.3000 "Possibilités de remplacer le système actuel de gestion en matière d'admission de médecins» et, l'été prochain, il devrait mettre en consultation un projet de loi pour piloter les médecins du secteur ambulatoire.

Les différentes formes de pilotages discutées au Parlement risquent de produire plus d'effets indésirables que d'effets souhaités, à l'image des limites supérieure et inférieure définies au niveau national pour encadrer le pilotage cantonal du nombre de médecins de l'ambulatoire. Cette première variante accentuerait encore un peu plus les conflits d'intérêts (déjà coûteux) des cantons qui subventionnent les prestations ambulatoires hospitalières. De plus, les patients ne s'arrêtent pas aux frontières cantonales mais s'orientent en fonction de la configuration régionale ou des grands centres urbains. Enfin, le nombre de médecins n'est plus aujourd'hui une mesure pertinente face aux nouveaux modèles de travail, à l'activité à temps partiel et à la vie de famille. Les équivalents temps plein seraient beaucoup mieux adaptés, mais pour cela - comme aussi pour beaucoup d'autres dimensions - les bases statistiques nécessaires font défaut.

La deuxième variante, basée sur des rémunérations différenciées en fonction de la région - une sorte de "supplément Préalpes» - ne répond pas à la réalité du terrain. Les exemples de cabinets installés aux limites cantonales, qui demeurent malgré une valeur du point tarifaire plus basse, démontrent que les différences doivent dépasser $20 \%$ pour être attractives et, par conséquent, elles seraient trop chères. De plus, ces prix devraient rester stables longtemps afin de correspondre aux cycles d'investissements des cabinets médicaux, faute de quoi les coûts augmenteraient, sans amélioration de la couverture en zone rurale.

La troisième variante, qui vise un assouplissement de l'obligation de contracter, révèle davantage une prise d'influence des organisations faîtières des assurances- maladie qu'un objectif ‘Qualité pour l'ambulatoire`: le libre choix du médecin serait obligatoirement limité, les critères financiers des caisses viendraient s'immiscer dans la relation médecin-patient et provoqueraient des transferts vers les secteurs hospitaliers non concernés par l'obligation de contracter.

Notre proposition se concentre sur quatre critères de qualité pertinents, cumulatifs, et faciles à implémenter sur l'ensemble du territoire. Ces critères permettent non seulement de réguler les admissions de manière qualitative mais aussi de répartir les spécialisations médicales en fonction des besoins:

- Activité médicale clinique d'au moins trois ans dans un établissement de formation postgraduée reconnu dans la discipline visée pour l'admission: cela permet-

Pour les admissions, la FMH propose quatre critères de qualité pertinents, cumulatifs, et faciles à implémenter sur l'ensemble du territoire.

trait d'éviter des admissions disproportionnées dans certaines spécialités, en s'appuyant sur le nombre restreint de postes cliniques disponibles dans ces domaines.

- Compétences linguistiques dans une des langues officielles de la région d'activité, attestées par un examen de langue passé en Suisse avant de débuter l'activité médicale: près de $40 \%$ des candidats étrangers échouent à ce niveau en Allemagne.

- Etudes de médecine équivalentes aux standards européens d'au moins 5500 heures d'enseignement théorique et pratique.

- Attestation de la formation continue en cours. Grâce au diplôme de formation continue, elle peut être facilement vérifiée dans toutes les 46 disciplines.

La mise en œuvre de ces quatre critères, avec en parallèle l'encouragement ciblé de postes d'assistanat au cabinet dans les régions ayant une faible densité médicale, permettrait d'arriver à une solution adaptée, sans ajouter de tâches administratives supplémentaires. La FMH se tient volontiers à disposition pour faire profiter de son expérience dans ces domaines et tend la main pour une solution orientée qualité. 